\title{
Awake resection of a left operculo-insular low-grade glioma guided by cortico-subcortical mapping
}

\author{
Thiébaud Picart, MD, ${ }^{1}$ and Hugues Duffau, MD, PhD ${ }^{1-3}$ \\ 1'Department of Neurosurgery, Gui de Chauliac Hospital, Montpellier University Medical Center, Montpellier; ${ }^{2}$ Institute for Neuro- \\ science of Montpellier, INSERM 1051, Team "Plasticity of Central Nervous System, Human Stem Cells and Glial Tumors," Saint \\ Eloi Hospital, Montpellier University Medical Center, Montpellier; and ${ }^{3}$ University of Montpellier, France
}

A 30-year-old right-handed female medical doctor experienced generalized seizures. MRI showed a left operculo-insular low-grade glioma. Awake resection was proposed. During the cortical mapping, counting and naming task combined with right upper limb movement enabled the identification of the ventral premotor cortex and negative motors areas. The so-called Broca's area was not eloquent. Subpial dissection was performed by avoiding coagulation until the inferior fronto-occipital fasciculus and the junction between the output projection fibers and the anterior part of the superior longitudinal fasciculus III were reached. The patient resumed a normal familial and socio-professional life despite the resection of Broca's area.

The video can be found here: https://youtu.be/OALkOtvctQw.

KEYWORDS awake surgery; brain mapping; direct electrical stimulation; low-grade glioma; white matter tracts; video

\section{Supplemental Information}

Please see accompanying transcript and references. 